\title{
The Fornax Deep Survey (FDS) with VST
}

\section{Optical properties of the dwarf galaxies in the Fornax cluster (Corrigendum)}

\author{
Aku Venhola ${ }^{1,2}$, Reynier Peletier ${ }^{2}$, Eija Laurikainen $^{1}$, Heikki Salo $^{1}$, Enrichetta Iodice ${ }^{3}$, Steffen Mieske ${ }^{4}$, \\ Michael Hilker ${ }^{5}$, Carolin Wittmann ${ }^{6}$, Maurizio Paolillo ${ }^{7,3}$, Michele Cantiello ${ }^{8}$, Joachim Janz ${ }^{1,13}$, Marilena Spavone ${ }^{3}$, \\ Raffaele D'Abrusco ${ }^{9}$, Glenn van de Ven ${ }^{14}$, Nicola Napolitano ${ }^{3}$, Gijs Verdoes Kleijn ${ }^{2}$, Massimo Capaccioli ${ }^{10}$, \\ Aniello Grado $^{3}$, Edwin Valentijn ${ }^{2}$, Jesús Falcón-Barroso ${ }^{11,12}$, and Luca Limatola ${ }^{3}$ \\ 1 Astronomy Research Unit, University of Oulu, Pentti Kaiteran katu 1, 90014 Oulu, Finland \\ e-mail: aku.venhola@oulu.fi, avenhola@student.oulu.fi \\ 2 Kapteyn Institute, University of Groningen, Landleven 12, 9747 AD Groningen, The Netherlands \\ 3 INAF - Astronomical Observatory of Capodimonte, Salita Moiariello 16, 80131 Naples, Italy \\ ${ }^{4}$ European Southern Observatory, Alonso de Cordova 3107, Vitacura, Santiago, Chile \\ 5 European Southern Observatory, Karl-Schwarzschild-Strasse 2, 85748 Garching bei München, Germany \\ 6 Astronomisches Rechen-Institut, Zentrum für Astronomie der Universität Heidelberg, Mönchhofstraße 12-14, \\ 69120 Heidelberg, Germany \\ 7 University of Naples Federico II, C.U. Monte Sant'Angelo, Via Cinthia, 80126 Naples, Italy \\ 8 INAF Osservatorio Astronomico d'Abruzzo, Via Maggini, 64100 Teramo, Italy \\ 9 Smithsonian Astrophysical Observatory, 60 Garden Street, 02138 Cambridge, MA, USA \\ 10 University of Naples Federico II, C.U. Monte Sant'Angelo, Via Cinthia, 80126 Naples, Italy \\ 11 Instituto de Astrofisica de Canarias, C/ Via Láctea s/n, 38200 La Laguna, Spain \\ 12 Depto. Astrofisica, Universidad de La Laguna, C/ Via Láctea s/n, 38200 La Laguna, Spain \\ 13 Finnish Centre of Astronomy with ESO (FINCA), University of Turku, Väisäläntie 20, 21500 Piikkiö, Finland \\ 14 Department of Astrophysics, University of Vienna, Türkenschanzstrasse 17, 1180 Wien, Austria
}

A\&A, 625, A143 (2019), https://doi .org/10.1051/0004-6361/201935231

Key words. galaxies: clusters: individual: Fornax - galaxies: dwarf - galaxies: evolution - galaxies: interactions galaxies: luminosity function, mass function - errata, addenda

This is an erratum for the paper "The Fornax Deep Survey (FDS) with VST VI. Optical properties of the dwarf galaxies in the Fornax cluster". We noticed two mistakes in Fig. 21 of the original paper that have a slight effect on the shown ram pressure stripping radii, but do not change conclusions of that figure. In addition, in the original paper, we had calculated cluster-centric distances of objects incorrectly in some parts of our analysis, which slightly affects the appearance of several figures in the original paper. We show here the corrected versions of all the figures that were affected by this mistake. In this Letter, we use original numbering of the figures to avoid confusion. The conclusions and findings of the original paper are not altered by these mistakes.

The first mistake in Fig. 21 of the original paper is that wrong velocities were used when estimating the stripping radii of the ram pressure stripping. This error was caused due to the first author's experiments of how the different cluster-centric velocities of the infalling galaxy affect the stripping radii. The results of these tests were mistakenly saved to the figure that appears in the published version. In the left panel of Fig. 21, we show the ram pressure stripping radii when using those velocities stated in the original text and legend.

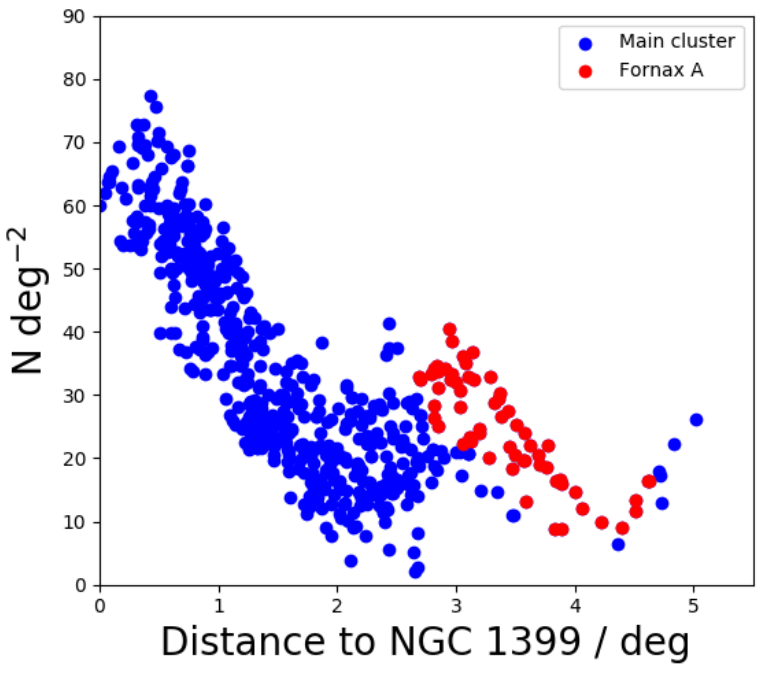

Fig. 4. Galaxy surface density as a function of distance from NGC 1399. The red points show the galaxies projected within one degree of the NGC 1316, which is the central galaxy of Fornax A group. The blue points show the other galaxies. 

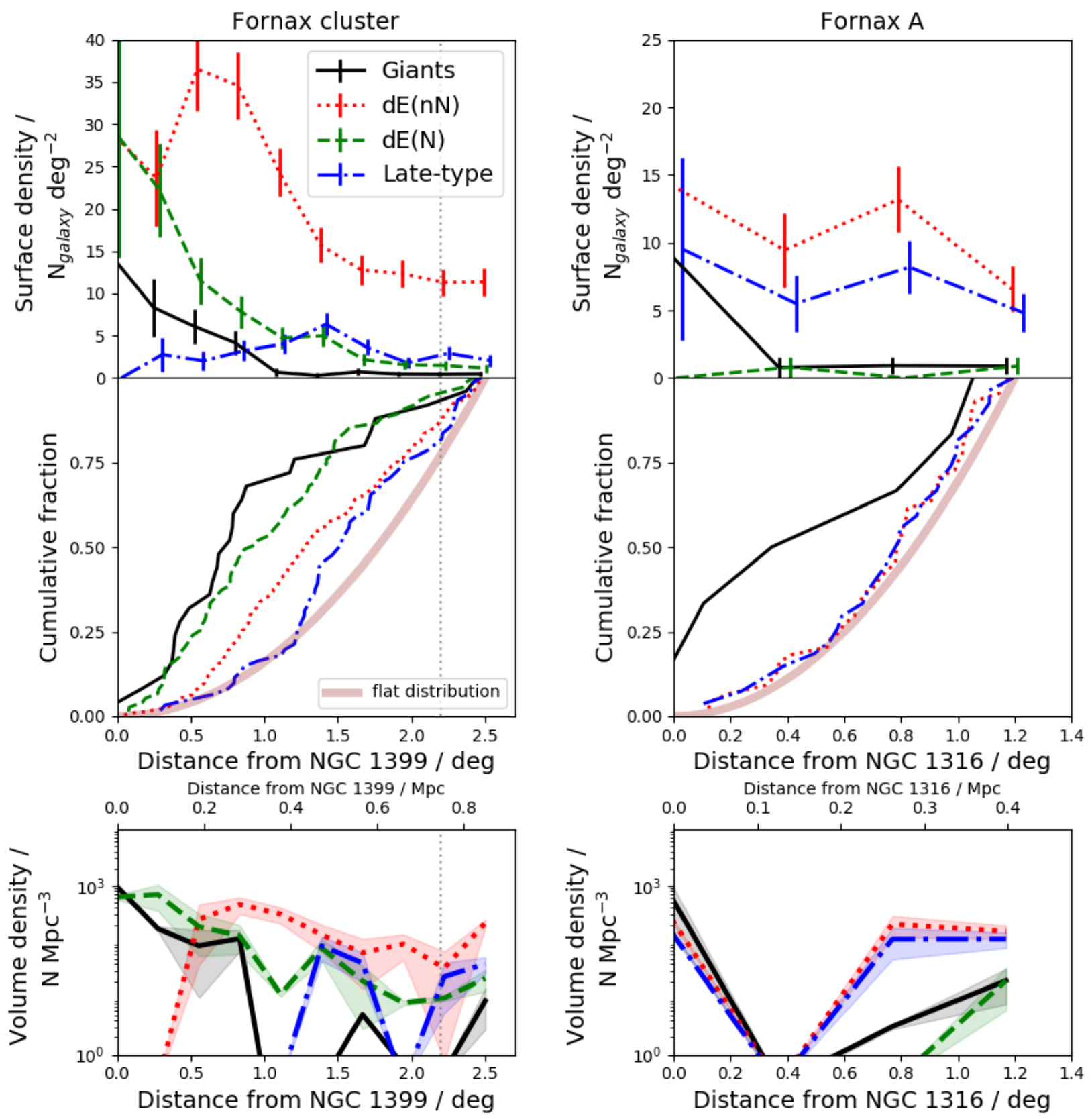

Fig. 5. Upper panels: surface number density of galaxies as a function of the cluster-centric radius (left panels), and distance from Fornax-A (right panels). Middle panels: corresponding cumulative number fractions as function of radii. The black solid lines correspond to the giant galaxies with $M_{r^{\prime}}<-18.5 \mathrm{mag}$, the green dashed and red dotted lines correspond to $\mathrm{dE}(\mathrm{N}) \mathrm{s}$ and $\mathrm{dE}(\mathrm{nN}) \mathrm{s}$, respectively, and the blue dash-dotted lines correspond to the late-type dwarfs. For a reference, the purple opaque lines show the cumulative profile for a uniform radial surface-density distribution without any cluster-centric concentration. Bottom panels: deprojected volume densities of the galaxies obtained from the radial surface density profiles using the onion peeling technique described in Appendix B. The shaded areas correspond to the uncertainties in the deprojected profiles, estimated with the Monte Carlo method. The vertical gray dotted lines in the left panels correspond to the virial radius of the cluster.

The second mistake regards the units of the middle panel of the original Fig. 21. The values are not in kpc as stated in the legend, but in degrees. In Fig. 21, we show the correct values in $\mathrm{kpc}$ and the updated disruption radii.

Correcting the first of these mistakes changes the order of the dotted and solid lines in Fig. 21 into the right order: for galaxies that have higher cluster-centric velocities the ram pressure stripping radii are shorter than for those with lower velocities. Additionally, the solid lines that correspond to the lower cluster-centric velocities are shifted approximately $0.5 \mathrm{dex}$ towards lower masses. In practice this means that the minimum mass of the galaxies that can have bound gas in their center when they move through the cluster is 0.5 dex lower than stated in the paper. This discrepancy does not change the conclusions of the original paper as the difference caused by this error is small. Additionally, these calculations are order of magnitude estimates and the velocity of individual galaxies has a significant effect to these estimates.

The second of the mistakes in the original Fig. 21 concerning the units in the middle panel is merely a typo in the legend, and thus does not change any aspects of the discussion. In the original paper, we had wrongly calculated Euclidean cluster-centric 


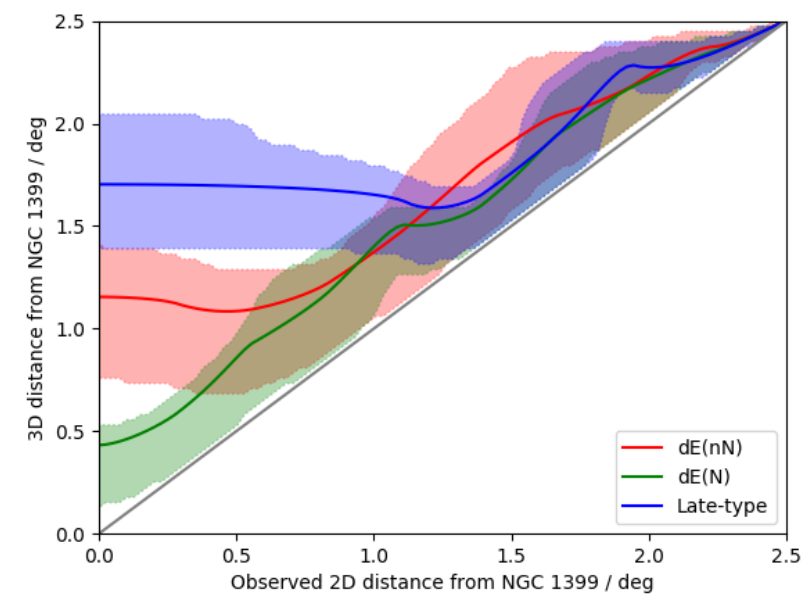

Fig. 6. Relations between projected $2 \mathrm{D}$ distances and likely $3 \mathrm{D}$ distances for the different morphological types. The green, red, and blue lines correspond to $\mathrm{dE}(\mathrm{N}), \mathrm{dE}(\mathrm{nN})$, and late-type dwarfs. The shaded areas show the range within the $25 \%$ and $75 \%$ in the probability distributions.
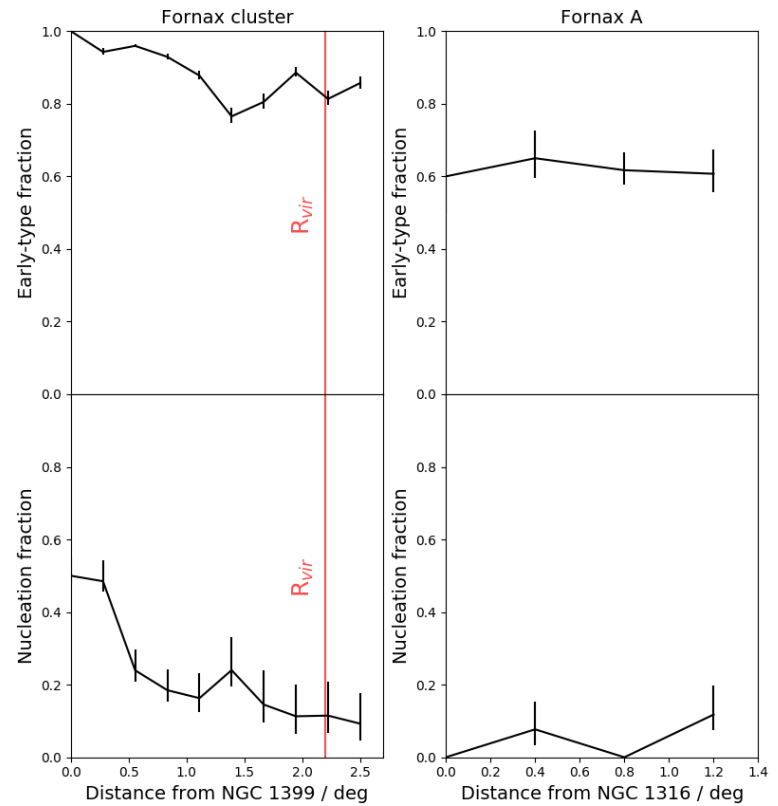

Fig. 7. Left panels: early-type fraction (upper left) and nucleation fraction (lower left) as a function of projected 2D distance from central galaxy NGC 1399 of the Fornax cluster. Right panels: same relations as a function of 2D distance from central galaxy NGC 1316 of the Fornax A sub-group. The red horizontal lines in the left panels show the virial radius of the main cluster.
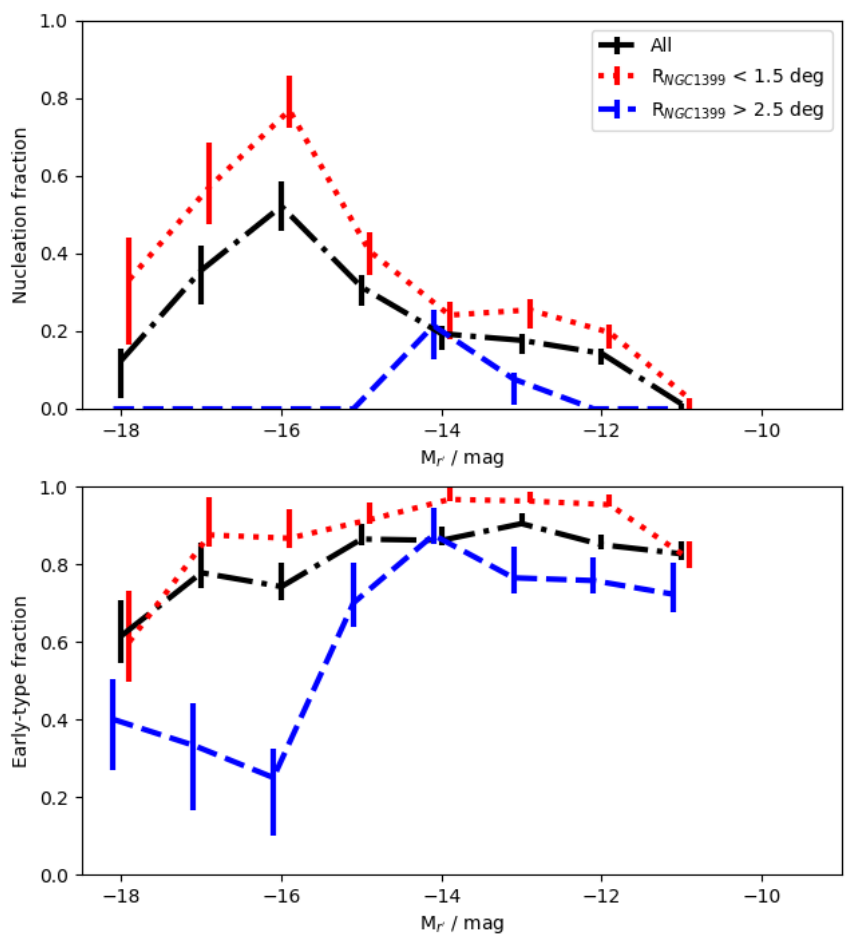

Fig. 9. Nucleation fractions as a function of absolute $r^{\prime}$-band magnitude at different cluster-centric radii. The red dotted, blue dashed, and black dash-dotted lines show the nucleation fraction within $1.5 \mathrm{deg}$ and outside $2.5 \mathrm{deg}$ from the cluster center, and for all galaxies, respectively.

distances from the object coordinates. This mistake causes errors of $0-20 \%$ to the cluster-centric distances, depending of the object's location, and it affects original Figs. 4-7, 9-11, 15, and 17. This mistake also affects Table F.1 of the original paper, which tabulates the values of Fig. 15. Here, we show the corrected versions of these figures. The corrected figures are otherwise identical to the original figures, but have been remade using the right distances. Correcting this mistake in the calculation of the cluster-centric distances slightly changes the curves and values in the above mentioned figures, but does not alter their overall trends and findings. The most remarkable change happens in the blue curves of Fig. 9. As few nucleated early-type dwarfs are shifted inwards from the outermost distance bin after correcting the distances, the early-type and nucleation fractions of that bin are lowered significantly from the high-luminosity end.

\section{References}

Roediger, J. C., Ferrarese, L., Côté, P., et al. 2017, ApJ, 836, 120 

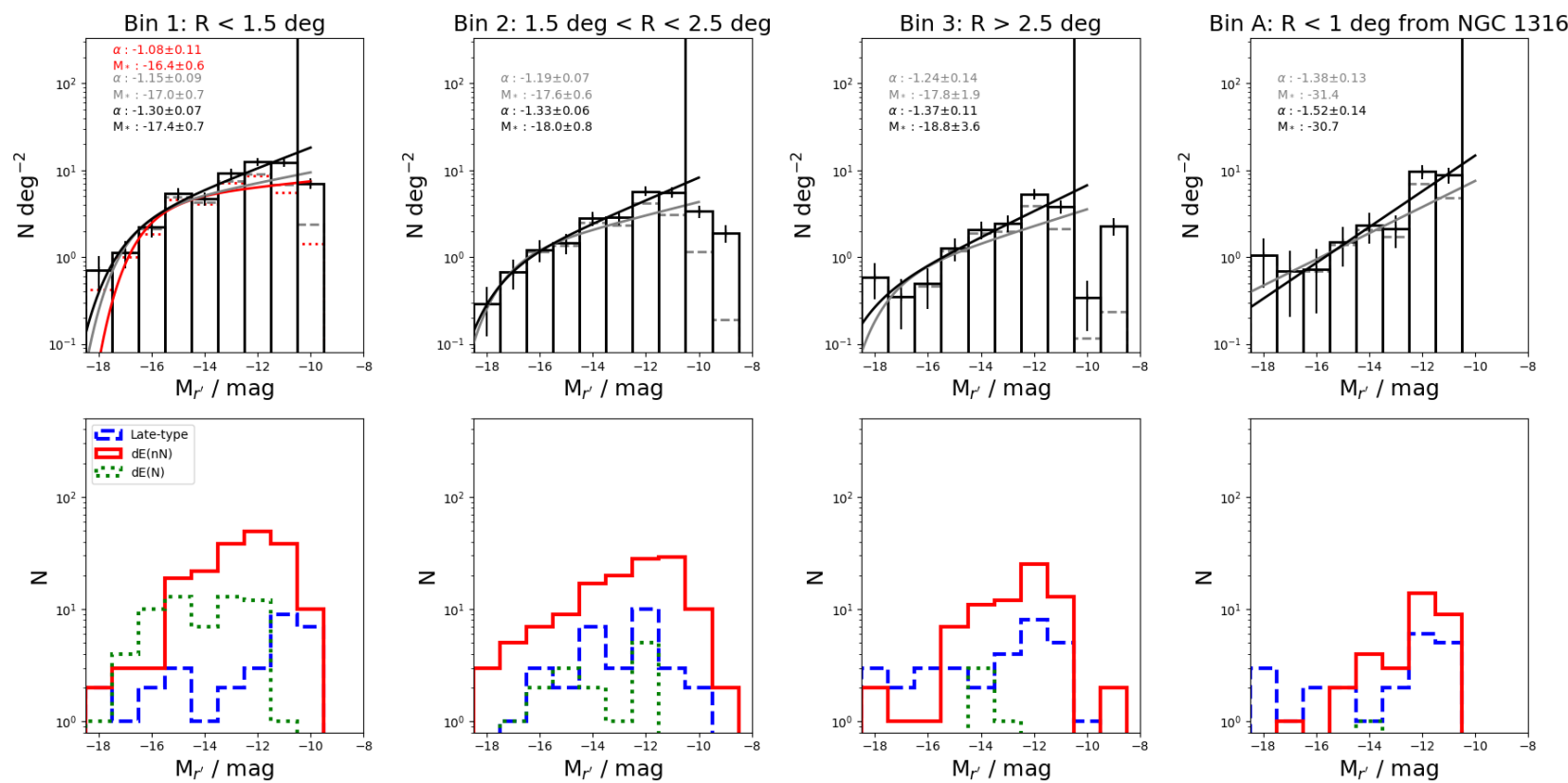

Fig. 10. Four upper panels: luminosity function in different spatial bins around the Fornax main cluster and the Fornax A group. At the distance of the Fornax cluster one degree corresponds to $350 \mathrm{kpc}$. The gray dashed histograms show the observed number of galaxies, and the black histograms show the numbers after correcting for the completeness. The black vertical lines indicate the $50 \%$ completeness limit of our catalog. The gray and black curves shows the fitted Schechter function for the observed and completeness corrected luminosity functions, respectively, and the red line in the upper left panel shows the fit for the innermost bin, when the late-type galaxies are excluded from the fit. The fit parameters of each bin are shown in the upper left corner of the panels with the colors corresponding to those of the fitted functions. Lower panels: luminosity distribution for the different types of galaxies in the bins.

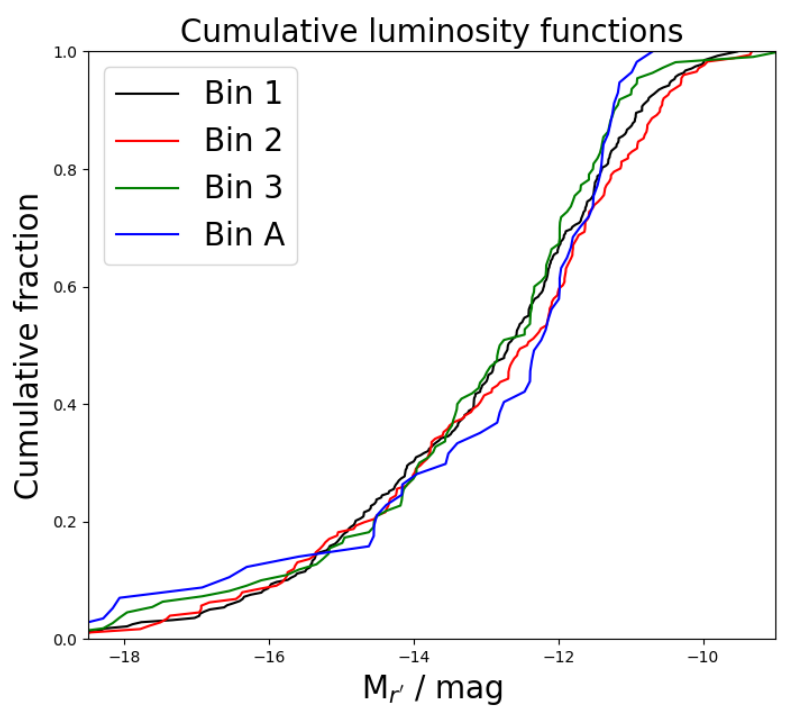

Fig. 11. Cumulative luminosity functions in bins defined in Fig. 10. 


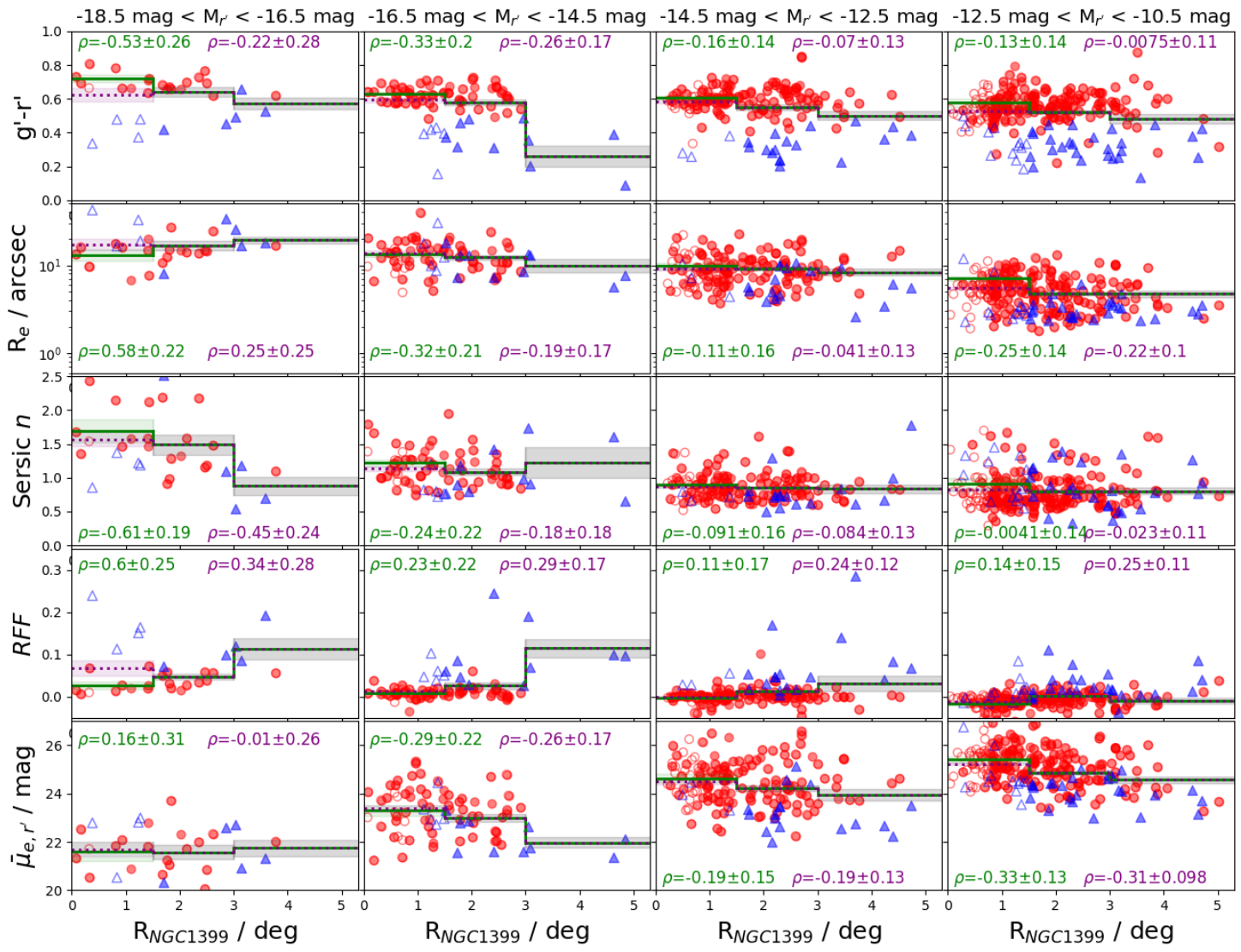

Fig. 15. Panel rows from top to bottom: $g^{\prime}-r^{\prime}$ color, effective radius $\left(R_{\mathrm{e}}\right)$, Sérsic index $(n)$, Residual Flux Fraction (RFF), and mean effective surface brightness $\left(\bar{\mu}_{\mathrm{e}, r^{\prime}}\right)$ of the Fornax dwarf galaxies as a function of their cluster-centric distance. The columns correspond to different luminosity bins. The lines and shaded areas show the bin means and uncertainties of the mean for the parameters. The green and purple colors correspond to the values when the projection effects are taken in account $(\mathrm{dE}(\mathrm{nN}) \mathrm{s}$ and late-types excluded from the inner parts as found in Sect. 3.2), and when raw data is used, respectively. The late-type and early-type dwarfs are shown with the blue and red symbols, respectively. The unfilled symbols correspond to the galaxies excluded from the inner parts in order to minimize the projection bias. The $\rho$ parameter indicates Spearman's correlation coefficient for the correlation of the parameter with distance, and its uncertainty.
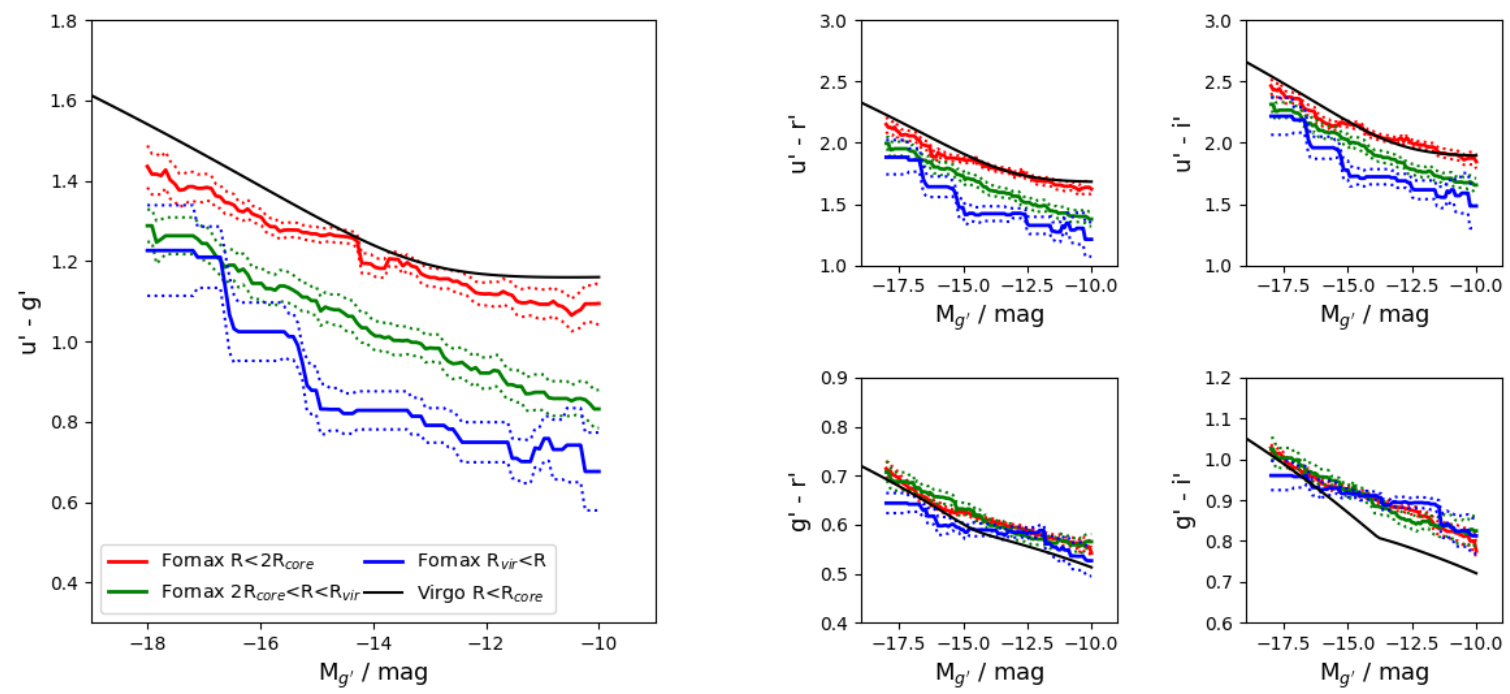

Fig. 17. Running means of the color-magnitude relations in different colors for the dEs. The red, green, and blue lines corresponds to inner, middle and outer bins in the Fornax cluster, respectively. The black lines show the Roediger et al. (2017) color-magnitude relation in the core of the Virgo cluster. We use the $g^{\prime}$-band absolute magnitudes on the $x$-axis instead of $r^{\prime}$-band, since the analysis for the Virgo was done using $g^{\prime}$-band. 

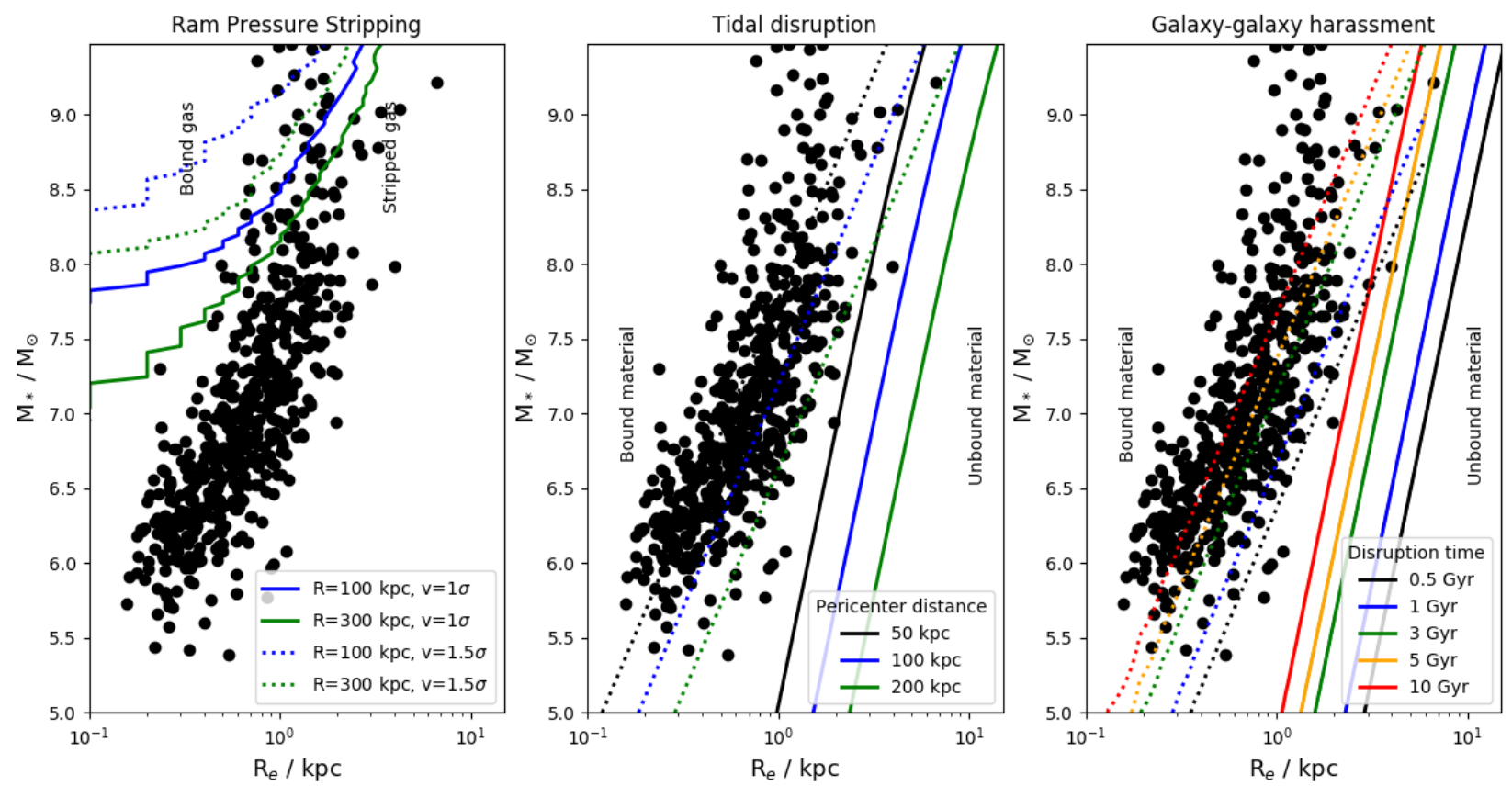

Fig. 21. Effectiveness of different environmental processes in the galaxy stellar mass-effective radius plane. The black points correspond to the FDSDC galaxies. The lines in the left panel show the stripping radius as a function of galaxy's stellar mass. For the areas on the right side of the lines, $\Pi_{\text {gal }}<P_{\text {ram }}$, and thus all the gas gets stripped. The blue and green lines show the stripping radii at the cluster-centric distances of 100 and $300 \mathrm{kpc}(\approx 0.3 \mathrm{deg}$ and $1 \mathrm{deg})$, respectively, and the solid and dotted lines correspond to galaxy velocities of $370 \mathrm{~km} \mathrm{~s}^{-1}$ and $550 \mathrm{~km} \mathrm{~s}^{-1}$, respectively. The lines in the middle panel show the relation between the tidal radius and galaxy mass for three orbits with different peri-center radii. The solid and dotted lines show the tidal radii for the galaxies when assuming DM-halo and without DM-halo, respectively. The solid and dotted lines in the right panel show the disruption times against harassment for the given mass and effective radius galaxies, when assuming a dark matter halo, and when using only the stellar mass for the calculation, respectively. 
A. Venhola et al.: The Fornax Deep Survey (FDS) with VST (Corrigendum)

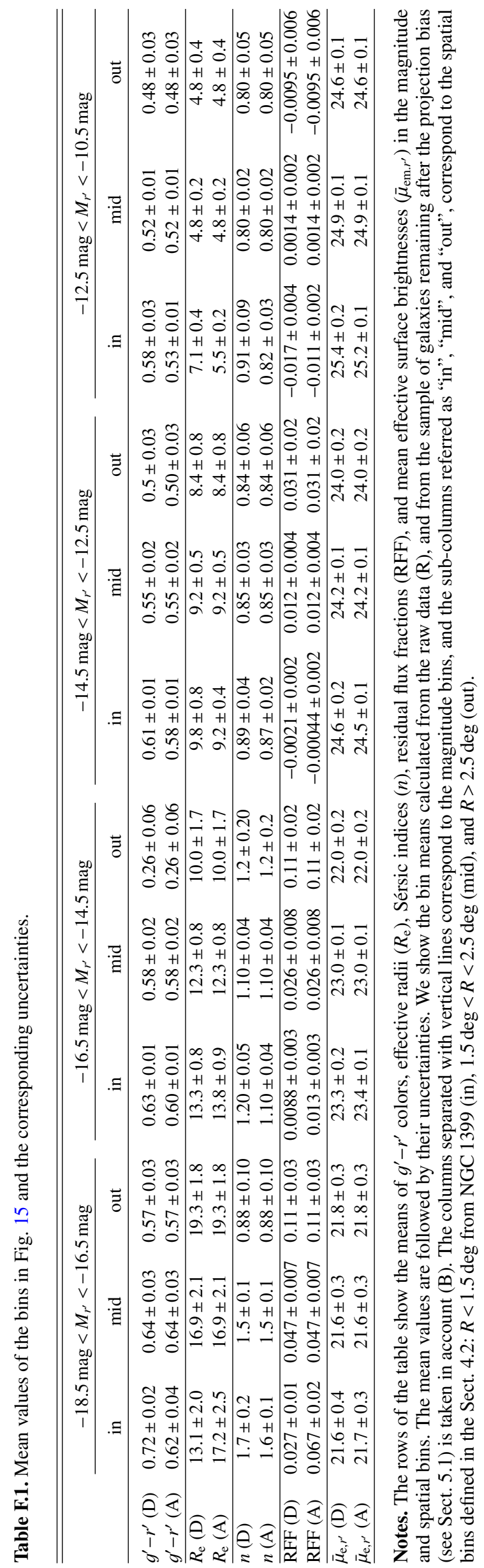

